\title{
Influencia del Flujo de Nitrógeno en la Estructura, Dureza y Resistividad de Recubrimientos de TiSiNO
}

\section{Leandro García ${ }^{1}$, Julián Hernández ${ }^{2}$, Claudia O. Mendoza ${ }^{1}$ y María G. Garnica ${ }^{3}$}

(1) Univ. Veracruzana, Centro de Investigación en Micro y Nanotecnología, Calzada Adolfo Ruiz Cortines 455, Fracc. Costa Verde, 94292 Boca del Río, Veracruz-México (e-mail: leagarcia@uv.mx) (2) Univ. del Papaloapan, Campus Loma Bonita, Av. Ferrocarril s/n, Colonia Cd. Universitaria, 68400 Loma Bonita, Oaxaca-México

(3) Univ. Michoacana de San Nicolás de Hidalgo, Facultad de Ingeniería Mecánica, Santiago Tapia 403 col. Centro, CP 58000, Morelia, Mich., México

\section{Resumen}

Recubrimientos de TiSiNO fueron fabricados por la técnica de erosión catódica reactiva por corriente directa sobre sustratos de acero y silicio, utilizando un blanco de Ti y una pequeña placa de Si sobre una atmósfera reactiva de nitrógeno, oxigeno y argón. Usando el método de Warren-Averbach se analizaron los cambios estructurales de las muestras en función de la variación del flujo de nitrógeno. Cálculos estequiométricos, obtenidos de la técnica de espectroscopia de energía dispersada y corroborados por espectroscopia de descarga luminiscente, muestran que los recubrimientos se encuentran en la región de solución sólida de $\operatorname{TiSi}_{x} \mathrm{~N}\left(\mathrm{O}_{y}\right)$ y $\operatorname{SiN}_{x} \mathrm{O}_{y}$, dominando la fase del oxinitruro de titanio. La muestra fabricada sobre sustratos de acero y silicio a un flujo de nitrógeno de $3 \mathrm{sscm}$, presentó el valor de dureza más alto y el valor de resistividad más bajo, respectivamente, debido a su estructura, orientación cristalina preferencial y fases cristalinas presentes en ella.

\section{Influence of Nitrogen Flow on the Structure, Hardness and Resistivity of TiSiNO Coatings}

\begin{abstract}
Coatings of $\mathrm{TiN}$ and $\mathrm{TiSiN}$ were prepared by reactive direct current magnetron co-sputtering on steel and on silicon substrates, using a Ti target and a small Si plate in a reactive atmosphere of nitrogen, oxygen and argon. Using the Warren-Averbach method the structural changes were analyzed in function of the variation of the nitrogen flow. Stoichiometry calculations obtained by X-ray Energy Dispersive Spectroscopy and corroborated by Glow Discharge Spectroscopy, show that the TiSiNO coatings contain two solid solutions of $\mathrm{TiSi}_{x} \mathrm{~N}\left(\mathrm{O}_{y}\right)$ and $\mathrm{SiN}_{x} \mathrm{O}_{y}$ and have a strong domain on titanium oxynitrure phase. The sample made on steel and silicon wafers substrates with nitrogen flow of 3 sscm, presented the highest hardness and the lowest resistivity values, respectively, due to its preferential crystalline orientation, structure and crystalline phases present.
\end{abstract}

Keywords: TiSiNO coatings, hardness, resistivity, $X$ ray diffraction, sputtering 


\section{INTRODUCCIÓN}

El estudio y desarrollo de la ciencia es fundamental para satisfacer la constante demanda de la sociedad en productos nuevos y de alta calidad. En este respecto, a medida que en la construcción de circuitos integrados se alcanzaban tamaños cada vez más pequeños y aumentaban el nivel de empaquetamiento, las interconexiones de aluminio comenzaron a presentar problemas y se comenzó a estudiar la posibilidad de utilizar cobre en lugar de aluminio como metal de interconexión debido a su resistencia a la electromigración y a su baja resistividad $(1.68 \mu \Omega \mathrm{cm})$. Sin embargo, el uso de este metal presenta algunos inconvenientes como su difusividad, ya que este elemento presenta una elevada movilidad en el silicio, que puede llegar a afectar y degradar seriamente las propiedades tanto del silicio como del óxido de silicio. Toda esta problemática puede solucionarse con el uso de capas de barrera, también llamadas barreras de difusión. Estas capas se sitúan alrededor de las interconexiones de cobre, tanto para evitar el contacto con otras capas metálicas o semiconductoras, como para evitar reacciones con el medio en los contactos externos. En general, los materiales elegidos para este fin son metales refractarios u sus nitruros, tales como TiN, TaN y TiZrN (Yeh et al., 2008; Lee y Kuo, 2007), dado que poseen estabilidad térmica y buenas propiedades eléctricas. De igual manera se ha encontrado que sistemas ternarios como el de TiSiN (Ee et al., 2006a; Watanabe et al., 2003), WSiN (Fleming et al., 1998; Nakajima et al., 1997), TaSiN (Letendu et al., 2006; Lee et al., 1999) ó ZrSiN (Zhang et al., 2007) son capaces de sustituir al TiN (Bonitz et al., 2005 y Cheng et al., 2005) en la fabricación de dispositivos dieléctricos como barreras de difusión, debido al mezclado de su microestructura, el cual se ha encontrado una notable mejora en sus propiedades, debido principalmente a la formación de una fase nanocristalina embebida en una matriz amorfa, generalmente de nitruro de silicio (Gao et al., 2004; Procházka et al., 2004).

Recientemente se han fabricado películas de TiSiNO para que actúen como barreras contra la difusión (Ee et al., 2006b, 2006c, 2006d; Shalaeva et al., 1999), debido a sus prometedoras propiedades, que los hacen fuertes candidatos para la siguiente generación de barreras de difusión en la tecnología CMOS. Estudios realizados sobre este sistema cuaternario, revelan que estos recubrimientos están formados de nanocristales embebidos generalmente en fases amorfas de Ti-O, Si-O, Si-N y Si-N-O (Shalaeva et al., 1999). Shalaeva desarrollo un estudio muy completo acerca de la composición de fases (diagrama metaestable) de estas películas de Ti-Si-N-O (Shalaeva et al., 1999), el cual es formado a partir del diagrama de equilibrio de los sistemas Ti-Si-N y Ti-Si-O. Otros autores se han enfocado a estudiar la resistividad de dicho sistema cuaternario en función del efecto que producen los parámetros de fabricación sobre su microestructura, morfología superficial, rugosidad y tipos de enlace químico (Ee et al., 2006b, 2006c, 2006d). Sin embargo, un estudio referente a su dureza, aun no ha sido reportado y mucho menos comparado con su resistividad. Por ello, este trabajo se enfoca en el análisis de la dureza de recubrimientos de TiSiNO en conjunto con sus valores de resistividad, la cual se relaciona con su estructura y composición química, para ver el efecto que produce en dicho sistema cuaternario fabricado por la técnica de erosión catódica reactiva por corriente directa sobre sustratos de acero y silicio.

\section{PROCEDIMIENTO EXPERIMENTAL}

Los recubrimientos de TiSiNO fueron fabricados utilizando un sistema de erosión catódica reactiva por corriente directa (dc), asistido por magnetrón, modelo (Intercovamex -sputtering V3) sobre substratos de acero y substratos de silicio tipo-p con un valor de resistividad de $5 \times 10^{5} \mu \Omega \mathrm{cm}$. Para ello, se utilizo un blanco de $\mathrm{Ti}$ y dos pequeñas fracciones de área de $\mathrm{Si}$, empleando una potencia de $200 \mathrm{~W}$ durante $2 \mathrm{hr}$. El sistema esta provisto de flujometros, los cuales permiten controlar con exactitud el flujo de los gases utilizados (argón, nitrógeno y oxigeno). Utilizando una bomba mecánica y una bomba turbomolecular, se logró que el sistema alcanzara un vació de $1 \times 10^{-6} \mathrm{mbar}$ y a través de termopares, la temperatura de sustrato empleada fue controlada en $400^{\circ} \mathrm{C}$ sobre sustratos de silicio y acero. La presión de trabajo fue de $8.5 \times 10^{-3}$ mbar y fue constante para todas las muestras obtenidas. Para obtener una buena adherencia entre el recubrimiento y el sustrato, se realizó primero una pre-erosión durante unos $10 \mathrm{~min}$., esto con el fin de remover contaminantes adsorbidos sobre la superficie del blanco, cubriendo a la vez al sustrato con un obturador. Posteriormente, se remueve el obturador y se deposita durante 15 min una capa metálica de Ti-Si 
(debido a que aproximadamente el $80 \%$ es de titanio). Esta capa tiene el objetivo de proveer de una interfase metálica entre el oxi-nitruro y el sustrato, lo que permite lograr una buena adherencia del recubrimiento de TiSiNO. Posteriormente se inició el crecimiento de los recubrimientos, introduciendo un flujo de una mezcla de nitrógeno, oxigeno y argón. Utilizando una temperatura de sustrato de $400^{\circ}$ C (valor de temperatura aproximado al cual se oxida el TiN) y un flujo constante de oxigeno de 10 $\mathrm{sccm}$, se logró oxidar los recubrimientos, generando el sistema cuaternario de Ti-Si-N-O. Se fabricaron cuatro conjuntos de muestras, las cuales se etiquetaron como M1, M2, M3 y M4. En dichas muestras el flujo de gas de argón fue mantenido constante en $25 \mathrm{sccm}$ y se varió el flujo de gas de nitrógeno en 3, 5, 7 y 9 sccm correspondiente a M1, M2, M3 y M4.

La estructura y parámetros cristalinos de los recubrimientos fueron determinados por difracción de rayos $X(D R X)$, utilizando un difractómetro marca RIGAKU, modelo DMAX, trabajando con la línea $\mathrm{Cu}-\mathrm{K} \alpha_{1}$ con longitud de onda de $1.5406 \AA$ A. La composición química de los recubrimientos fue determinada por espectroscopia de energía dispersiva (EED) usando un microscopio electrónico de barrido (MEB), marca PHILIPS XL30 ESEM. De igual manera, las longitudes de las diagonales de las huellas de indentación Vickers fueron medidas por MEB. Las pruebas de dureza fueron llevadas acabo utilizando un microdurometro Vickers modelo BRUEHLER Micromet 2100, aplicando cargas de $0.098,0.245,0.49,0.98,1.96$ and $2.94 \mathrm{~N}$. Por otro lado, las mediciones de resistividad fueron realizadas por el método de van der Pauw.

Finalmente la composición química de los recubrimientos en función de su profundidad, fue determinada por medio de un espectrofotómetro de descarga luminiscente (GDS) utilizando para ello un sistema LECO Glow Discharge Spectroscopy modelo GDS850A. Cabe mencionar que la mayoría de las caracterizaciones fueron realizadas sobre sustratos de acero excepto las de resistividad, donde el sustrato empleado fue el silicio. Lo anterior con la finalidad de medir sus propiedades mecánicas y de resistividad en los sustratos que normalmente son empleados en las aplicaciones industriales.

\section{RESULTADOS Y DISCUSIONES}

La Figura 1 muestra los patrones de difracción de rayos $X$ del conjunto de cuatro muestras fabricadas a diferentes flujos de nitrógeno sobre substratos de acero. El pico de difracción correspondiente a la primer muestra (M1) presenta un pico en $2 \theta=44.73^{\circ}$, el cual corresponde al acero que se utilizó como sustrato. Las muestras presentan fases cristalinas con una orientación preferencial (220), excepto la muestra M2 que presenta una orientación preferencial (200). La orientación cristalina (220), es más eficiente que la (111) y (200), ideal para poder utilizarse en recubrimientos como barreras para los circuitos integrados (Ee et al., 2006a, 2006b), garantizando así el éxito de los dispositivos electrónicos. No obstante, esta orientación cristalina preferencial no es tan fácil de obtener, ya que la estructura columnar del TiN, presenta la orientación cristalina preferencial (111) ó (200). De igual manera en la Figura 1, se puede observar que la intensidad de los picos de difracción de las muestras va disminuyendo conforme el flujo del gas de nitrógeno va aumentado, donde las fases cristalinas presentes corresponden a fcc-TiN (JADE PDF-card \# 381420) y fcc-(TiO) $)_{3.38}$ (JADE PDF-card \# 75-0310). En estos patrones de difracción se puede observar una fuerte dependencia de la textura de las películas con respecto al flujo de nitrógeno, así como un corrimiento en los picos de difracción conforme el flujo de nitrógeno va incrementándose, trayendo como consecuencia una transición de una fase fcc-TiN a una fase fcc- $(\mathrm{TiO})_{3.38}$, debido a los esfuerzos intrínsecos generados por la variación del flujo de nitrógeno.

El método de Warren-Averbach (McKeehan y Warren, 1953) fue utilizado para analizar el perfil de línea de los picos de difracción, donde los parámetros obtenidos se listan en la Tabla 1. El tamaño de grano cristalino para la fase de fcc-TiN va disminuyendo conforme el flujo de gas de nitrógeno se va incrementando, hasta que esta fase se transforma en una fase completamente amorfa. Este incremento en el flujo de nitrógeno, provoca a su vez, que el tamaño cristalino de la fase cristalina fcc- $(\mathrm{TiO})_{3.38}$ vaya en aumento y se estabilice en un valor de $5.5 \mathrm{~nm}$, donde la fase cristalina fcc-TiN ha desaparecido por completo. Así mismo, las variaciones en el ancho de la función representado por el ancho del pico a media altura (FWMH) son atribuidas a la variación del tamaño de grano promedio y a microdeformaciones. 


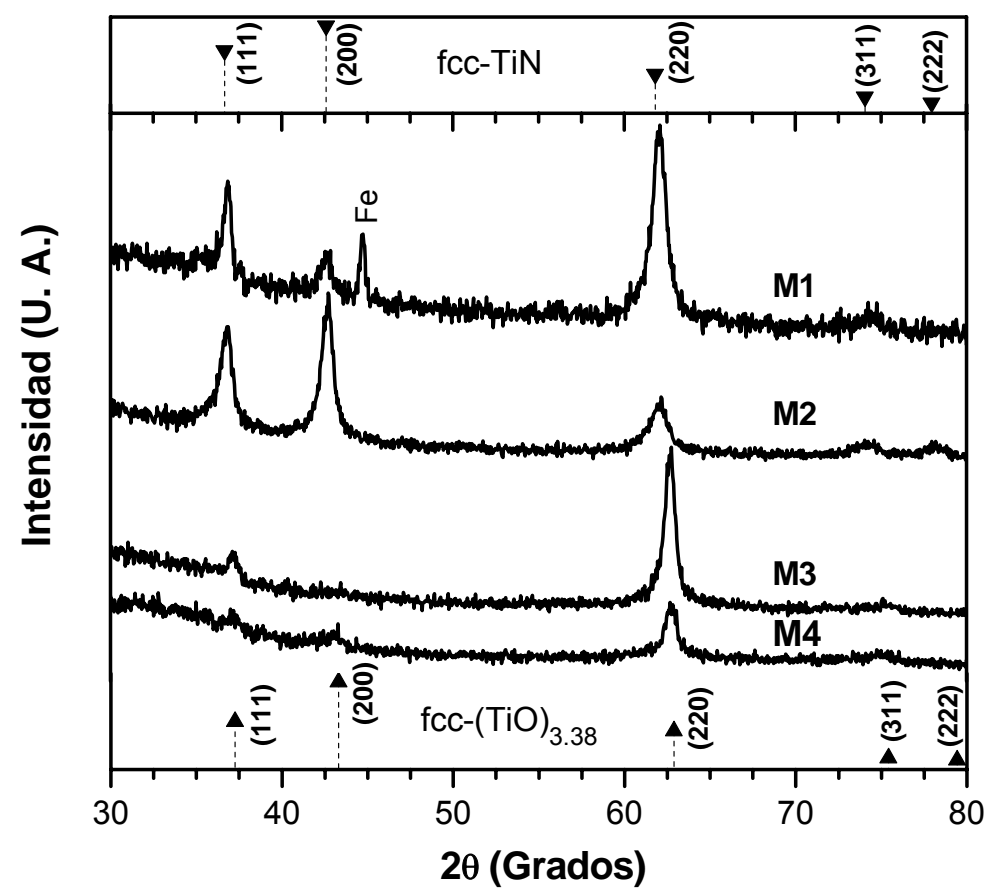

Fig. 1: Patrón de difracción de rayos $\mathrm{X}$ de muestras crecidas a diferentes flujos de nitrógeno y a una temperatura de sustrato de $400^{\circ} \mathrm{C}$.

Tabla 1: Parámetros obtenidos a partir del método de Warren-Averbach.

\begin{tabular}{|c|c|c|c|c|c|c|c|}
\hline \multirow[b]{2}{*}{ Recubrimiento } & \multirow[b]{2}{*}{$\begin{array}{c}\text { Flujo de } \\
\text { nitrógeno } \\
\text { (sccm) }\end{array}$} & \multicolumn{3}{|c|}{ TiN } & \multicolumn{3}{|c|}{$(\mathrm{TiO})_{3.38}$} \\
\hline & & $\begin{array}{c}2 \theta_{(200)} \\
\text { (grados) }\end{array}$ & $\begin{array}{c}\text { FWHM } \\
\text { (grados) }\end{array}$ & $\begin{array}{c}\text { Tamaño } \\
\text { de } \\
\text { grano } \\
\text { (nm) }\end{array}$ & $\begin{array}{c}2 \theta_{(200)} \\
\text { (grados) }\end{array}$ & $\begin{array}{c}\text { FWHM } \\
\text { (grados) }\end{array}$ & $\begin{array}{c}\text { Tamaño } \\
\text { de } \\
\text { grano } \\
(\mathrm{nm})\end{array}$ \\
\hline M1 & 3 & 62.077 & 0.974 & 5.2 & $\begin{array}{l}-- \\
--\end{array}$ & --- & --- \\
\hline M2 & 5 & 61.420 & 2.556 & 3.0 & 62.104 & 1.059 & 5.8 \\
\hline M3 & 7 & 62.741 & 1.300 & 4.3 & 62.695 & 0.538 & 11.8 \\
\hline M4 & 9 & --- & --- & --- & 62.796 & 0.874 & 5.5 \\
\hline
\end{tabular}

La composición química de cada muestra fue obtenida por EED, empleando las muestras depositadas sobre los sustratos de acero, para evitar cualquier cuantificación errónea del contenido de silicio en ellas, encontrándose arriba de un 25 At \% de nitrógeno en todas ellas. La composición química de cada película en porcentaje atómico fue de $\mathrm{Ti}_{39.36} \mathrm{Si}_{7.46} \mathrm{~N}_{29.04} \mathrm{O}_{24.14}, \mathrm{Ti}_{37.21} \mathrm{Si}_{9.51} \mathrm{~N}_{27.56} \mathrm{O}_{25.72}$, $\mathrm{Ti}_{33.98} \mathrm{Si}_{7.9} \mathrm{~N}_{40.59} \mathrm{O}_{17.53}$ y $\mathrm{Ti}_{30.35} \mathrm{Si}_{9.75} \mathrm{~N}_{38.90} \mathrm{O}_{21}$, para $\mathrm{M} 1, \mathrm{M} 2$, M3 y M4 respectivamente. Difracción de rayos $X$ no da información explicita respecto del silicio, sin embargo EED si lo detecta, lo cual, de acuerdo a investigaciones reportadas en la literatura (Kim et al., 2002b; Vaz et al., 2002), este elemento podría estar presente en estas películas, como una fase amorfa de $\mathrm{Si}_{3}(\mathrm{~N}, \mathrm{O})_{4}$ (Pilloud et al., 2008; Shalaeva et al., 1999). Estos resultados concuerdan con la estequiometría obtenida en los recubrimientos por EED para formar $\mathrm{Si}_{3}(\mathrm{~N}, \mathrm{O})_{4}$. Por otro lado, la fase de nitruro de titanio puede generalmente ser depositada con una alta cristalinidad a baja temperatura con la ayuda de la energía del plasma, pero los cerámicos como el nitruro de silicio y la alumina, necesitan una alta temperatura para cristalizar, debido a su alta viscosidad (Kim et al., 2002a).

Los valores de dureza fueron obtenidos a partir del modelo de indentación de trabajo, propuesto por Korsunsky et al. (1998), ilustrándose el comportamiento obtenido para cada muestra en la Figura 2. El diagrama propuesto por Korsunsky, representa esquemáticamente la evolución que va sufriendo la dureza respecto al cambio de profundidad de indentación relativa provocada por la aplicación de diferentes cargas. Korsunsky en este diagrama muestra que la plasticidad del recubrimiento domina a bajas cargas, pero cuando la carga es aumentada, existe mayor penetración, y por lo tanto, el recubrimiento llega a fracturarse. Para el análisis de dureza de las películas de TiSiNO, se aplicaron 
las siete cargas más bajas del durómetro Vickers, obteniendo un comportamiento en forma de "rodilla", donde la curva tiende al valor de dureza del sustrato para cargas grandes, mientras que para cargas pequeñas, la curva tiende hacia el valor de dureza real del recubrimiento. Este comportamiento obtenido, esta por encima de la décima parte del espesor del recubrimiento, lo que significa que el valor de la dureza obtenido es influenciado por una contribución grande del sustrato. El valor de la dureza del sustrato de acero empleado es de $1.5 \mathrm{GPa}$, valor que el modelo de indentación de trabajo aproxima a $1.66 \mathrm{GPa}$. Esto significa que los valores de dureza obtenidos para las muestras M1, M3 y M4 son prácticamente el doble del valor del sustrato y para la muestra M2 es el triple. Para eliminar esta contribución y obtener valores de dureza más cercanos al real, es necesario aplicar cargas más bajas.
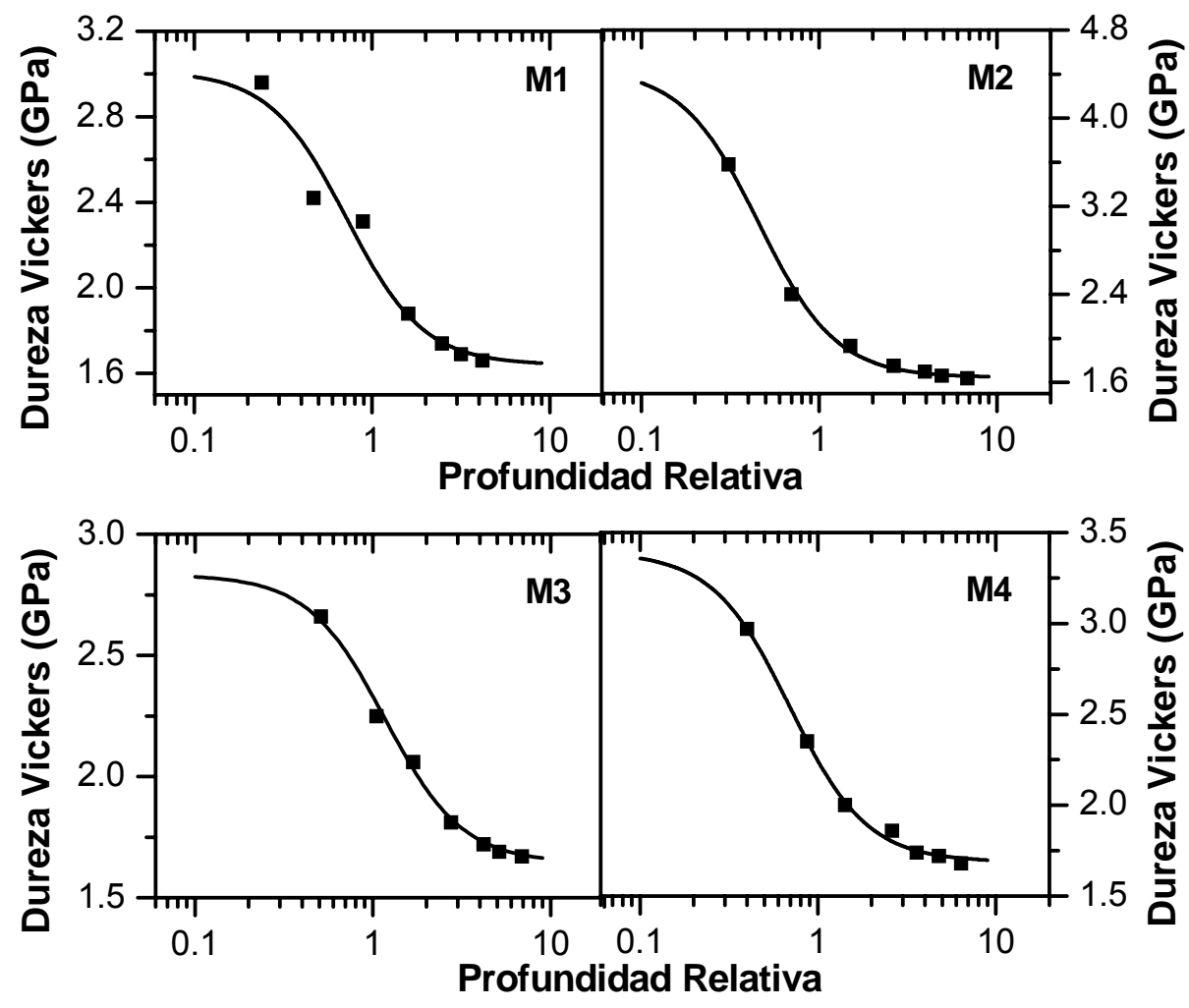

Fig. 2: Comportamiento tipo "rodilla" de las muestras obtenidas empleando el método de indentación de trabajo.

En la Figura 3 se muestra el perfil de concentración como función de la profundidad en rangos variables que van desde 0 hasta $5 \mu \mathrm{m}$. Se puede apreciar claramente el comportamiento de cada uno de los elementos de las capas interiores que conforman cada una de las muestras. Esta técnica corrobora los resultados obtenidos por EED, debido a que se detectan los cuatro elementos depositados en todas las muestras (titanio, silicio, nitrógeno y oxigeno), los cuales fueron cuantificados durante todo el análisis. El sistema GDS comienza a detectar arriba del boro, lo que garantiza la detección de los cuatro elementos que se manejan. El perfil de composición en profundidad para el silicio en todas las muestras es de intensidad muy baja, pero con la cantidad suficiente en los recubrimientos para ser significativa. Las mediciones de GDS fueron realizadas hasta detectar el sustrato de acero, por lo cual el intervalo que se muestra en profundidad en la Figura 3 varía desde 3 hasta $5 \mu \mathrm{m}$. De igual manera, estas mediciones proporcionan el espesor de cada uno de los recubrimientos. Los respectivos espesores de los recubrimientos de TiSiNO son 3.4, 1.8, 2.6 y $1.8 \mu \mathrm{m}$, correspondientes a M1, M2, M3 y M4, respectivamente. El depósito de los recubrimientos se realizó de manera homogénea, garantizando una buena eficiencia y poca contaminación, lo cual puede ser determinante en el control de calidad para una aplicación dada. Por ultimo, cabe aclarar que las intensidades que se muestran en el eje vertical de la Figura 3 no son equivalentes a las cantidades en por-ciento en peso o atómico que deba existir de cada elemento, sino que es solamente una guía que permite identificar la evolución del elemento en las capas internas del material. 

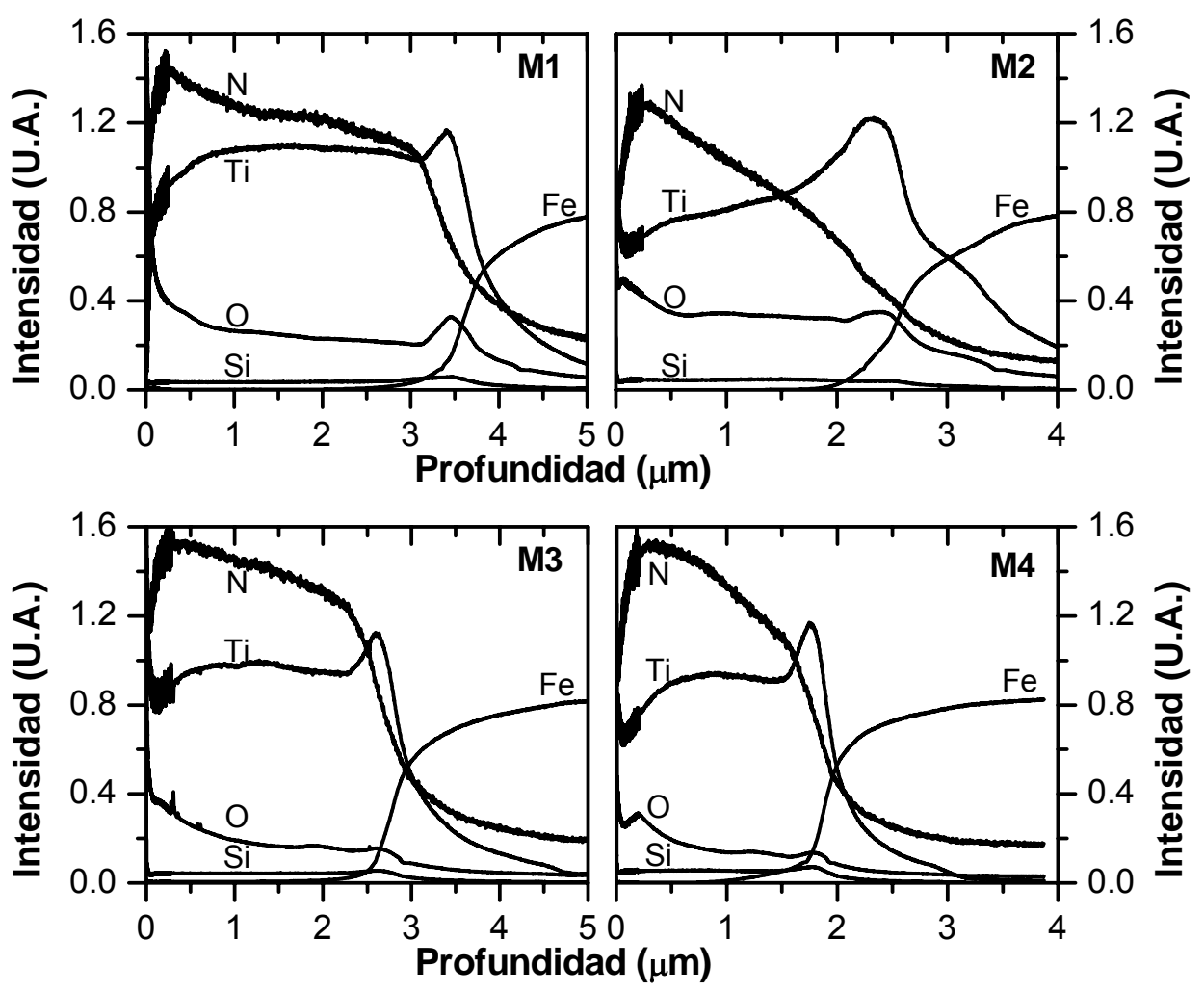

Fig. 3: Perfil de concentración de las muestras obtenidas como función de su profundidad.

Por otro lado, utilizando el método de van der Pauw, se calcularon los valores de resistividad de cada una de las muestras obtenidas sobre substratos de silicio. Del análisis realizado, se encontró que el recubrimiento con la dureza más alta $(4.4 \mathrm{GPa})$, muestra $\mathrm{M} 2$, presenta el menor valor de resistividad $\left(3.36 \times 10^{5} \mu \Omega \mathrm{cm}\right)$, resultado que coincide con el hecho de que esta muestra presenta la estructura columnar del TiN, con orientación cristalina preferencial de (200) y con el coeficiente de textura relativo más alto para el pico (200). A pesar de que no es la muestra con menor tamaño de grano para la fase TiN, si lo es para la fase fcc- $(\mathrm{TiO})_{3.38}$.

Por otro lado, mientras las muestras restantes presentan una disminución en su valor de dureza (por debajo de los $3.4 \mathrm{GPa}$ ), su valor en resistividad aumenta considerablemente (desde $2.27 \times 10^{6} \mu \Omega \mathrm{cm}$ para M3 hasta $1.02 \times 10^{8} \mu \Omega \mathrm{cm}$ para M1), comportamiento atribuido a que estas muestras presentan la orientación cristalina preferencial (220). Este resultado revela que el contenido de nitrógeno es muy importante para determinar la dureza de las películas de TiSiNO. Por consiguiente el tamaño de grano no es el único parámetro que hay que considerar para explicar las variaciones de la dureza de las películas de este tipo de sistemas cuaternarios, debido a que tanto la estructura, como la fase presente y la composición química de cada película son factores muy importantes a tomar en cuenta.

\section{CONCLUSIONES}

Se estudió el efecto de la variación del flujo de nitrógeno sobre la dureza, estructura y composición química de los recubrimientos de TiSiNO fabricados por la técnica de erosión catódica reactiva por corriente directa. Para un flujo de nitrógeno de $5 \mathrm{sccm}$, se logró obtener la estructura columnar del TiN, con orientación cristalina preferencial (200). Esta orientación cristalina es una de las típicas para que el fcc-TiN arroje como resultado un valor de dureza mayor. La muestra con mayor dureza, resultó ser M2 y por la orientación cristalina preferencial, resulto ser la muestra con menor valor de resistividad. Por el contrario, para las demás muestras, el valor de resistividad fue alto, mientras que el valor de dureza fue bajo. Concluyendo así que los recubrimientos de TiSiNO son notablemente afectados por el flujo de nitrógeno que es empleado para su fabricación, sobre todo, para la aplicación que se les quiera dar. 


\section{AGRADECIMIENTOS}

Este trabajo fue financiado por CONACYT (Proyecto no. 58653). Los autores agradecen el apoyo técnico de J.E. Urbina Álvarez, M.A. Hernández Landaverde, P.G. Jiménez, R. Flores Farías, y de R. Juárez Aguirre.

\section{REFERENCIAS}

Bonitz, J., R. Ecke, S.E. Schulz y T. Gessner; Different $\mathrm{SiH}_{4}$ Treatments of CVD TiN Barrier Layers, Microelectron Eng.: 82(3-4), 618-622 (2005).

Cheng, H.-E., W.-J. Lee y C.-M. Hsu; The Effect of Deposition Temperature on the Properties of TiN Diffusion Barriers Prepared by Atomic Layer Chemical Vapor Deposition, Thin Solid Films: 485(1-2), 59-65 (2005).

Ee, Y.C. y otros cinco autores; Copper Diffusion in Ti-Si-N Layers Formed by Inductively Coupled Plasma Implantation, Appl. Surf. Sci.: 253(2), 530-534 (2006a).

Ee, Y.C. y otros 9 autores; Bias-Temperature Stability of Ti-Si-N-O Films, J. Electrochem. Soc.: 153(5), G470-G474 (2006b).

Ee, Y.C., Z. Chen, S.B. Law y S. Xu; Formation and Characterization of Ti-Si-N-O Barrier Films, Thin Solid Films: 504(1-2), 218-222 (2006c).

Ee, Y.C. y otros 4 autores; Low Temperature Physical-Chemical Vapor Deposition of Ti-Si-N-O Barrier Films, Electrochem. Solid-State Lett.: 9(3), G100-G103 (2006d).

Fleming, J.G. y otros siete autores; Growth and Properties of W-Si-N Diffusion Barriers deposited by chemical vapor deposition, Thin Solid Films: 320(1), 10-14 (1998).

Gao, L., J. Li, T. Kusunose y K. Niihara; Preparation and Properties of TiN-Si ${ }_{3} \mathrm{~N}_{4}$ Composites; J. Eur. Ceram. Soc.: 24(2), 381-386 (2004).

Kim, K.H., S.-R. Choi y S.-Y. Yoon; Superhard Ti-Si-N Coatings by a Hybrid System of Arc lon Plating and Sputtering Techniques, Surf. Coat. Technol.: 161(2-3), 243-248 (2002a).

Kim, S.H., S.H. Jang, S.S. Kang y K.H. Kim; Synthesis and Mechanical Evaluation of Nanocomposite Coating Layer of nc-TiN/a-Si ${ }_{3} \mathrm{~N}_{4}$ on SKD 11 Steel by Sputtering, J. of Mat. Process. Technol.: 130131(1), 283-288 (2002b).

Korsunsky, A.M., M.R. McGurk, S.J. Bull y T.F. Page; On the Hardness of Coated System, Surf. Coat. Technol.: 99(1-2), 171-183 (1998).

Lee, Y.-J., B.-S. Suh, M.-S. Kwon y C.-O. Park; Barrier Properties and Failure Mechanism of Ta-Si-N Thin Films for Cu Interconnection, J. Appl. Phys.: 85(3), 1927-1934 (1999).

Lee C. y Y.-L. Kuo; The evolution of Diffusion Barriers in Copper Metallization, JOM: 59(1), 44-49 (2007)

Letendu, F. y otros cinco autores; TaSiN Diffusion Barriers Deposited by Reactive Magnetron Sputtering, Thin Solid Films: 513(1-2), 118-124 (2006).

McKeehan, M. y B.E. Warren; X-Ray Study of Cold Work in Thoriated Tungsten, J. Appl. Phys.: 24(1), 52-56 (1953).

Nakajima, K. y otros cinco autores; Formation Mechanism of Ultrathin WSiN Barrier Layer in a WWN $N_{x} /$ Si System, Appl. Surf. Sci.: 117-118(1), 312-316 (1997). 
Pilloud, D., J.F. Pierson, M.C. Marco de Lucas y A. Cavaleiro; Study of the Structural Changes Induced by Air Oxidation in Ti-Si-N Hard Coatings, Surf. Coat. Technol.: 202(11), 2413-2417 (2008).

Procházka, J., P. Karvánková, M.G.J. Vepřek-Heijman y S. Vepřek; Conditions Required for Achieving Superhardness of $\geq 45 \mathrm{GPa}$ in $n c-T i N / a-\mathrm{Si}_{3} \mathrm{~N}_{4}$ Nanocomposites, Mater. Sci. Eng. A: 384(12), 102-116 (2004).

Shalaeva, E.V., S.V. Borisov, O.F. Denisov y M.V. Kuznetso; Metastable Phase Diagram of Ti-Si$N(O)$ Films $\left(C_{S i}<30\right.$ at.\%), Thin Solid Films: 339(1-2), 129-1336 (1999).

Vaz, F. y otros cinco autores; Young's Modulus of (Ti,Si)N Films by Surface Acoustic Waves and Indentation Techniques, Thin Solid Films: 408(1-2), 160-168 (2002).

Watanabe, H. y otros cinco autores; The Mechanical Properties and Microstructure of Ti-Si-N Nanocomposite Films by lon Plating, Surf. Coat. Technol.: 169-170(1), 452-455 (2003).

Yeh, T.-S., J.-M. Wu, L.-J. Hu; The Properties of TiN Thin Films Deposited by Pulsed Direct Current Magnetron Sputtering, Thin Solid Films: en prensa, (2008).

Zhang, H. y otros cuatro autores; The Effect of ZrSiN Diffusion Barrier on the Bonding Strength of Titanium Porcelain, Surf. Coat. Technol.: 201(9-11), 5637-5640 (2007). 